\title{
Checkpoints, sauf-conduits et contrôle de la population en Égypte au début du VIII ${ }^{\mathrm{e}}$ siècle
}

\author{
Alain Delattre
}

1 Introduction*

Diverses sources, littéraires comme documentaires, écrites en grec, en copte et en arabe, nous renseignent sur les restrictions et entraves apportées à la libre circulation des personnes au début de l'époque arabe en Égypte. L'image qu' on peut en tirer est variée, voire contradictoire. L'objectif de cette contribution est de réexaminer les sources disponibles ainsi que des documents inédits, et de les comparer, afin de tenter de déterminer quels étaient concrètement les mécanismes de contrôle de la population.

Seront passés en revue l'Histoire des patriarches (2), les sauf-conduits (3), les garanties du monastère de Jérémias à Saqqarah (4), les "lettres de protection" (5) et les laissez-passer de la région thébaine (6). Ce dernier dossier s' est vu récemment complété par de nouveaux documents ou des textes connexes (7) dont l'étude permet de faire progresser l'état de la question.

\section{L'Histoire des patriarches}

Un passage célèbre de l'Histoire des patriarches d'Alexandrie décrit de manière vive et frappante les réglementations édictées en l'an 96 de l'Hégire, soit en 714/715, par le directeur des finances Usāma b. Zayd al-Tanūhī (en poste en 714717 et $720-723)$. La biographie du patriarche Alexandre II $\left(705^{-730}\right)^{1}$ relate ainsi les entraves à la circulation des personnes et les difficultés rencontrées pour obtenir un passeport, ou plus exactement un sauf-conduit ${ }^{2}$.

* Le présent article a été réalisé dans le cadre du projet "Christians in Egypt" mené à l' Université de Leyde et financé grâce à un Marie Curie Intra-European Fellowship for Career Development (projet PIE F-GH-2009-254782).

1 La source copte (perdue) à la base des Vies 43-46 aurait été rédigée vers 770 par un certain Jean $\mathrm{I}^{\mathrm{er}}$, et serait donc de peu postérieure aux événements. Je remercie vivement Perrine Pilette pour les informations qu' elle m'a fournies à ce propos.

2 Basil Evetts, "History of the Patriarchs of the Coptic Church of Alexandria: Agathon to 
For he [Usāma] wrote and said: "Wherever a man is found walking, or passing from one place to another, or disembarking from a boat, or embarking, without a passport, he shall be arrested, and the contents of the boat confiscated, and the boat burnt." And if any Romans were found on the river, they were brought to him; and some of them he slew, and others he impaled, and the hands and feet of some he cut off. At last the roads were made impassable, and no man could travel or sell or buy. The fruits of the vineyards were wasted, and there was no one to buy them for a single dirhem, because their owners remained within their houses for two months, awaiting the passport to release them thence. If a mouse ate a man's passport, or if it were injured by water or fire or any accident, whether part or the whole of it remained to his possession, if its lettering were damaged, it could not be changed for a new one until he paid five dinars as a fee for it, and then it could be changed for him.

trad. B. EVETTS

Le tableau est sans doute dramatisé, mais il témoigne d'un durcissement des mesures de contrôle de la population, qui s'inscrit dans le contexte de la politique omeyyade des années $710-720^{3}$. Les opérations militaires, surtout navales, lancées contre l' empire byzantin nécessitaient beaucoup d' argent, des matériaux et aussi des hommes; plus généralement, le contexte économique et politique de la première moitié du viII ${ }^{\mathrm{e}}$ siècle a contribué à renforcer le contrôle exercé sur la population ${ }^{4}$. La pression fiscale avait été accrue, nécessitant un examen plus strict des déplacements, que justifiait aussi la possible présence d'espions byzantins. Le texte nous dit donc qu'il était interdit de circuler sans sauf-conduit et il était long et coûteux d'en obtenir un. De tels documents n'étaient d'ailleurs peut-être pas toujours suffisants pour se prémunir d'une arrestation ${ }^{5}$.

Michael III (766)," Patrologia Orientalis 5, no. 1 (1910): 69-70. On notera, à la suite de Yūsuf Rāgoib, "Sauf-conduits d'Égypte omeyyade et abbasside," Annales Islamologiques 31 (1997): 145, qu'il est préférable de réserver le terme de passeport aux documents destinés à voyager hors d'Égypte.

3 Frank R. Trombley, "Sawīus Ibn al-Muqaffa' and the Christians of Umayyad Egypt: War and Society in Documentary Context," dans Papyrology and the History of Islamic Egypt, éds. Petra M. Sijpesteijn et Lennart Sundelin, (Leiden: Brill, 2004), 205-206.

4 Petra M. Sijpesteijn, Shaping a Muslim State:The World of a Mid-Eighth-Century Egyptian Offcial (Oxford: Oxford University Press, 2013), 115-216.

5 Cf. PERF 598, 9; cf. Werner Diem, "Einige frühe amtliche Urkunden aus der Sammlung Papyrus Erhzerzog Rainer (Wien)," Le Muséon 97 (1984): 146-150; Sijpesteijn, Shaping a Muslim State, 97 et texte 31 . 
Une quinzaine de sauf-conduits arabes, qui correspondent à ceux dont il est question dans le texte de l'Histoire des Patriarches, ont été publiés jusqu'à présent ${ }^{6}$. Ces documents sont appelés sijill dans les textes littéraires, c' est-àdire la transcription du grec $\sigma \gamma \gamma$ i $\lambda$ ı qui décrit la forme du document et non son contenu. Le même terme est utilisé dans les papyrus coptes et grecs, mais les sauf-conduits arabes sont usuellement désignés par le terme kitāb, «document ${ }^{7}$. Les exemplaires conservés datent des époques omeyyade et abbasside (717 pour le plus ancien, $750 / 75^{1}$ pour les plus récents ${ }^{8}$ ). Ils émanent du bureau du surintendant des finances sous les Omeyyades, et directement de celui du gouverneur sous les Abbassides.

À titre d' exemple, je reproduis ici la traduction de Y. Rāgib, légèrement modifiée, de P.Cair. IFAO Inv. Ar. Gr. Copte o31, daté de $734^{9}$. Le document a été écrit à Armant (Hermonthis) et contient un sauf-conduit, destiné à Samouêl, fils de Mouei, qui l' autorise à se rendre et travailler au Fayoum, pour une période de trois mois.

(En grec, un résumé) Samuel (?) Mouei jusqu'à Arsinoé. (En arabe) Au nom de Dieu, clément et miséricordieux! Ceci est une lettre de 'Ilqima

6 Rāğib, "Sauf-conduits d'Égypte;" Naïm Vanthieghem, "Le plus ancien sauf-conduit arabe," Der Islam 91, no. 2 (2014): 266-271; Perrine Pilette et Naïm Vanthieghem, "Un nouveau saufconduit du monastère d'Apa Jeremias à Saqqara?" Bulletin of the American Society of Papyrologists 53 (2016): 233-238. Sur ces documents, voir aussi Gladys Frantz-Murphy, Arabic Agricultural Leases and Tax Receipts from Egypt, 148-427 A.H./765-1035 A.D. (Vienna: Hollinek, 2001), 106-109 et Gladys Frantz-Murphy, "Identity and Security in the Mediterranean World ca. AD 640-ca. 1517," dans XXVth International Congress of Papyrology. Ann Arbor, July 29August 4, 2007, éd. Traianos Gagos, American Studies in Papyrology (Ann Arbor: University of Michigan Press, 2010), 253-264.

7 Le mot sijill est apparemment utilisé également pour désigner les sauf-conduits dans les textes arabes, cf. Sijpesteijn, Shaping a Muslim State, 242; Petra M. Sijpesteijn, "Coptic and Arabic Papyri from Deir al Balāizah," dans Actes du $26^{e}$ Congrès international de papyrologie, éd. Paul Schubert (Genève: Droz, 2012), 710-711; Vanthieghem, "Sauf-conduit arabe," 267-268, n. 7 .

8 P.Cambridge UL Inv. Michaelides A 112 date de 717, c' est-à-dire de la première surintendance de Usāma b. Zayd al-Tanūhī, celui-là-même qui est mentionné dans l'Histoire des Patriarches (Vanthieghem, "Sauf-conduit arabe"); P.Lond. BL Inv. Or. 15, P.Saqqara 94/27 A, P.Paris BN Inv. Ar. 4633 et 4634 datent de 750/751 (Rāgib, "Sauf-conduits d'Égypte," nos. V-VIII).

9 Rāgib, "Sauf-conduits d'Égypte," 152-153, no. III. La date du document n’ est pas conservée, mais la validité du laissez-passer s' étend du 10 février 734 au 9 mai 734. 
b. al-Ḥārith, l'agent de l'émir 'Ubayd Allāh b. al-Ḥabhāāb sur le nome d'Armant et ses districts ${ }^{10}$, [à Samue]l Mouei, jeune, [...] des gens de Qașr. [...] Je lui ai permis de [travailler] dans le district du Fayoum pour s'a[quitter] de ce qu'il doit et je lui ai fixé un délai de trois mois, du premier jour de la lune de muharram à la fin du mois de $r_{a b i ̄}$ I l' année cent seize. Qui donc le rencontrera des agents de l'émir ou autres (agents de l'État) ne devra lui montrer que du bien. Que le sa[lut soit sur celui qui suit la direction!]

On trouve des formules similaires dans deux documents grecs, P.Sijp. 25 et Chrest.Wilck. $24^{11}$. Dans P.Sijp. 25, le mieux conservé, un certain Hôriôn écrit à l'émir responsable des fugitifs pour lui annoncer qu' après avoir obtenu une garantie, il a donné à sept moines de la petra d'Apa Mêna, au sud d'Assiout, l' autorisation d' aller dans la pagarchie d'Heliopolis pour une durée de six mois. Hôriôn demande ensuite à l'émir de confirmer sa décision. On trouve à la fin du document la liste des moines, dont il est précisé, comme dans P.CLT 3 (voir ci-dessous), qu'ils sont libres, c'est-à-dire que ce ne sont pas des fugitifs. Il apparait que le document constituait à la fois un sauf-conduit en lui-même, mais qu'il s'agissait aussi d'une demande adressée à l'émir, comme l'indique le terme même qui décrit le document l. 12: $\pi \alpha \rho \alpha x \lambda \eta \tau i x o ̀ v ~ \gamma \rho \alpha ́ \alpha \mu \alpha$, "lettre de demande"12. Le papyrus Chrest.Wilck. 24 est moins bien conservé; il est défini comme un $\sigma r i \lambda_{\lambda}$ เov et avait été établi en grec et en arabe (cf. l. 9 et 10). Il pourrait bien s' agir ici de la partie grecque d'un sauf-conduit bilingue grec-arabe. L'existence de tels documents n'a pas encore été mise en évidence, mais elle ne serait pas étonnante: au début du vıII ${ }^{\mathrm{e}}$ siècle, de nombreux documents officiels, comme les entagia par exemple, étaient bilingues.

L'Histoire des Patriarches indique qu' obtenir un sauf conduit prenait du temps (jusqu'à deux mois), mais on ne sait pas exactement quelle procédure il fallait suivre, ni quelles garanties il fallait donner. Une lettre copte de la première moitié $\mathrm{du} \mathrm{VIII}^{\mathrm{e}}$ siècle, P.CLT 3, fournit quelques renseignements à ce propos. Dans cette missive adressée à l'émir, Khaêl et Jôhannês, du kastron de Djême, demandent un sauf-conduit pour trois moines d'un monastère de

\footnotetext{
10 La lecture hayyizihā (au lieu du hayrihā suggéré par l'éditeur) a été proposée dans Sijpesteijn, Shaping a Muslim State, 141, n. 135.

11 Les deux textes sont datés respectivement du 29 Pakhôn ou du 5 Pauni d'une $12^{\mathrm{e}}$ indiction, soit les 24 et 30 mai d'une $12^{\mathrm{e}}$ année; en suivant les arguments paléographiques des éditeurs, on peut proposer 698 ou 713, mais 728 ne semble pas a priori exclu. Janneke de Jong éditera prochainement un texte similaire de la collection de Wurtzbourg.

12 Cf. Fritz Mitthof, "Urkundenreferat 2007 (2. Teil)," Archiv für Papyrusforschung 55, no. 1 (2009): 162 .
} 
la région thébaine et s'en portent garants. Il est très probable que les expéditeurs de la lettre sont des fonctionnaires locaux; en suivant l' opinion de Walter C. Till, on peut proposer de les identifier avec deux administrateurs (dioikêtês) de Djême et dater le document de $728 / 729$ ou $743 / 744^{13}$. Je propose ci-joint une traduction des lignes $2-10^{14}$.

(...) Ces moines de la Jarre d'Apa Paulos, qui vous apporteront cette humble missive, ceux-là donc que nous décrirons à la fin de cette lettre (c'est-à-dire dans le compte noté à la fin du document), veulent aller au nord, dans le nome du Fayoum, pour vendre leur petite production de cordes, qui est le fruit de leur travail. Ils ne peuvent faire cela sans un sigillion de votre Seigneurie. Nous demandons donc à votre honorable Seigneurie d'ordonner qu' on leur donne un sigillion pour trois mois précisément, à partir d'aujourd' hui, afin qu'ils trouvent le moyen d'aller au nord pour vendre leur petite production de corde, qui est le fruit de leur travail et de leur vie. Ce sont aussi des hommes libres et voici la garantie que nous effectuons pour leurs personnes. Nous l' avons envoyée à votre Seigneurie. La paix avec vous par Dieu ... † Et ils ont donné ce qui leur échoit pour les impôts de la $12^{\mathrm{e}}$ année de l'indiction

Une liste des trois moines, écrite en grec, conclut le document; les moines y sont présentés et même décrits physiquement. Avec un tel document, les bureaux de l'émir pouvaient émettre un sauf-conduit du type de ceux conservés dans la documentation arabe. Un aspect de la procédure de l'obtention des sauf-conduits se laisse ainsi deviner : il fallait avoir des garants pour obtenir un laissez-passer de l' administration arabe.

Avant d'émettre un laissez-passer, l'administration exigeait donc des garanties: il s' agissait de s'assurer de la solvabilité du contribuable et d'obtenir que

\footnotetext{
13 Walter C.Till, Datierung und Prosopographie der koptischen Urkunden aus Theben (Vienne: Österreichische Akademie der Wissenschaften, 1962), 42, 68, 111 et 235. Le document a apparemment été écrit par le scribe Aristophanês, fils de Jôhannês (sur ce personnage, cf. Jennifer Cromwell, "Aristophanes Son of Johannes: An Eighth-Century Bilingual Scribe? A Study of Graphic Bilingualism," dans The Multilingual Experience in Egypt, from the Ptolemies to the Abbasids, éd. Arietta Papaconstantinou (Farnham: Ashgate, 2010), 221-232).

14 Cf. aussi la discussion dans Sofia Schaten, "Reiseformalitäten im frühislamischen Ägypten," Bulletin de la Société d'Archéologie Copte 37 (1998): 95-97.
} 
quelqu'un s'engage à payer pour lui s'il ne remplissait pas ses obligations fiscales. La garantie pouvait être incorporée dans la lettre par laquelle on demandait aux autorités d'émettre un sauf-conduit, comme dans le cas de P.CLT 3, ou pouvait constituer un document à part. Dix papyrus du monastère de Jeremias à Saqqarah illustrent cette dernière procédure ${ }^{15}$. Ces textes du viII ${ }^{\mathrm{e}}$ siècle sont écrits par des moines et adressés au supérieur du monastère: les moines s'y portent garants pour l' un d' entre eux afin que le supérieur lui donne un sigillion pour aller en "Égypte." Le terme de кнмє, "Égypte", peut avoir diverses acceptions et peut désigner la vallée du Nil, la Basse ou la Haute-Égypte, mais aussi l'Égypte par rapport à Alexandrie et à Fusțāt ${ }^{16}$. Dans le contexte, il me semble probable que l' expression signifie quitter le désert, c' est-à-dire le monastère. Je propose ici une traduction de $C P R$ IV 2O, 1-9 (= SB Kopt. II 919).

Tout d' abord, au nom de Dieu. Moi, Hêla, fils de Timothe et ..., fils de Klousane et Enôch, fils de Kamoul, moines d' apa Jeremias à Memphis, nous écrivons au père bien-aimé, apa Daueid, le prêtre, archimandrite et higoumène de ce même monastère: nous nous portons garants pour Abraham, fils de Nahroou, afin que tu lui donnes un sigillion pour l'Égypte. Nous nous portons garants pour son impôt ou toute chose qui lui incombera ensuite du dikaion de la diaconie. Pour ta sécurité, nous avons rédigé ce document et d' autres personnes sont témoins, à savoir ... [suivent les souscriptions des témoins et la date.]

Un point du texte est d'interprétation délicate: on voit les moines s'adresser au supérieur pour qu'il "donne" un laissez-passer. Cela signifie-t-il que les saufconduits pouvaient, dans certains cas au moins, être rédigés en copte par une autorité locale, dans ce cas monastique? Le fait serait étonnant, d' autant que six sauf-conduits arabes émis par l' administration sur les quinze exemplaires édités proviennent justement du même monastère de Jeremias ${ }^{17}$. Il faut plutôt imaginer que les garanties étaient destinées au supérieur, qui se chargeait ensuite de déposer une demande de laissez-passer auprès de l'administration arabe. Reste que la garantie pouvait peut-être, dans une certaine mesure, autoriser son porteur à se déplacer, comme on le voit dans les "lettres de protection".

\footnotetext{
15 Sofia Schaten, "Reiseformalitäten," 94-95.

16 Walter E. Crum, A Coptic Dictionary (Oxford: Clarendon Press, 1939), $110 a$.

17 Pilette et Vanthieghem, "Nouveau sauf-conduit," 234-235.
} 
Les "lettres de protection", Schutzbriefe en allemand, forment un corpus d' environ cent documents, datés généralement entre le VII ${ }^{\mathrm{e}}$ et le $\mathrm{IX}^{\mathrm{e}}$ siècle et provenant, en majorité, de la région thébaine ${ }^{18}$. Ils présentent tous l'expression 入огос мпмоүте, littéralement "parole de Dieu", qui désigne le document. La formule doit sans doute se comprendre, comme l'a indiqué Walter C. Till, comme une "garantie par Dieu"19. Ces textes sont écrits par des autorités locales, civiles (lachanes, dioikêtês, etc.) ou parfois religieuses (supérieurs de monastère), qui autorisent le porteur à circuler. Le plus souvent les documents s'adressent à des fugitifs qui obtiennent ainsi le droit de rentrer chez eux, comme par exemple dans P.Schutzbriefe 4.

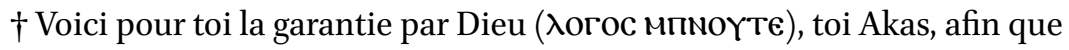
tu ailles et résides dans ta maison. Rien de mal ne t'atteindra. Moi, apa Biktôr, le lachane, je marque mon accord à cette garantie

L'aspect local du document apparaît immédiatement: aucun patronyme n' est mentionné, le texte n'est pas daté et on n'y trouve aucune mention de toponyme, ni pour indiquer où réside Akas, ni pour déterminer de quel village Biktôr est le lachane. De tels documents devaient permettre de se déplacer sur de courtes distances, mais ne constituaient pas, à mon sens, de réels sauf-conduits. Il s' agissait essentiellement d' une garantie: en cas de problème, c' est l' autorité qui avait délivré le document qui était selon toute vraisemblance tenue respon-

18 Aux documents rassemblés dans P.Schutzbriefe et Alain Delattre, "Les 'lettres de protection' coptes," dans Akten des 23. Internationalen Papyrologenkongresses. Wien, 22.-28. Juli 2001, éd. Bernhard Palme (Vienne: Verlag der Österreichischen Akademie der Wissenschaften, 2007), 173-178, on peut maintenant ajouter cinq nouveaux documents: P.Bawit Clackson 65 , P.Scholl 11 et 12, SB Kopt. III 1365 et 1367. La publication de documents encore inédits est annoncée, notamment quelques lettres de protection de la TT $115^{2}$ (cf. Iwona Antoniak, "Preliminary Remarks on the Coptic Ostraca from the Seasons 2003 and 2004," Polish Archaeology in the Mediterranean 16 (2005): 244-247) et un document d'Antinoé (P. Ant. Inv. 591 v., qui sera publié dans Alain Delattre et Rosario Pintaudi, "Les archives de Paule, fils de Petros, de la rue du Sauveur," dans Antinoupolis III, éd. Rosario Pintaudi (Florence: Istituto Papirologico "G. Vitelli," à paraître)). On notera aussi qu'il existe des documents similaires, écrits en grec, à l'époque byzantine, cf. P.Cair.Masp. I 6709o; sur ce document, voir aussi Sofía Torallas Tovar et Amalia Zomeño, "El control de la población en el Egipto pre y protoárabe," dans Historiografía y representaciones III: estudios sobre las fuentes de la conquista islámica, éds. Luis A. García Moreno, Esther Sánchez Medina et Lidia Fernández Fonfría (Madrid: Real Academia de la Historia, 2009), 617-618.

Sur le sujet, voir Delattre, "Lettres de protection." 
sable. À en juger par le nombre d'exemplaires conservés, ces petits déplacements, à l' intérieur d'un même nome sans doute, étaient fréquents, nettement plus que les voyages plus longs pour lesquels l'administration arabe exigeait sans doute un sauf-conduit en bonne et due forme.

Dans certains cas, les "garanties par Dieu" sont couplées à des reçus de taxe, comme par exemple P.Schutzbriefe $62^{20}$.

† Voici deux trimêsia comptés qui sont venus à moi, de ta part à toi, Zacharias, fils de Daueid, pour ton diagraphon de la première katabolê de la première année (de l'indiction). Total: 2/3 de sou. Hathyr, le 21; $7^{\mathrm{e}}$ année de l'indiction. Et voici la garantie par Dieu pour toi, pour ne pas te poursuivre pour aucun motif. Écrit au mois d'Hathyr, le $21 ; 7^{\mathrm{e}}$ année de l'indiction ...21

La garantie est ici fournie directement par le receveur. On notera par ailleurs que les reçus de taxe sur ostraca de la région thébaine - plus de 400 documents - apparaissent dans les années 710 avant de disparaître vers $730^{22}$. Il serait tentant de voir un lien entre les restrictions à la libre circulation et le changement de procédures administratives qui est à l'origine de ces reçus sur ostraca. On pourrait ainsi imaginer qu' une quittance pouvait servir de laissez-passer. Cependant, il semble que l'ostracon n' ait pas toujours été remis au contribuable (certains ont été archivés dans une administration) et surtout le reçu atteste le paiement d' une taxe, le plus souvent l'impôt personnel, mais ne fait pas le point sur la situation fiscale de l'individu ${ }^{23}$. La question de

20 On notera que les reçus de taxe arabes de l'époque abbasside contiennent parfois aussi des clauses qui assurent la libre circulation du contribuable, cf. Sijpesteijn, Shaping a Muslim State, 216, 240-241.

21 L'écart entre l'année d'imposition (première indiction) et la date de la rédaction (septième indiction) est considérable et de ce fait très suspect. Les points en dessous du chiffre $\zeta$ indiquent que la lecture du chiffre est incertaine. Le document a très vraisemblablement été écrit par le scribe Psate, fils de Pisraêl; il date sans doute de la fin des années 710, c' està-dire le moment où Psate rédige les reçus de taxe.

22 Paul E. Kahle, "Zu den koptischen Steuerquittungen," dans Festschrift zum 150-jährigen Bestehen des Berliner Ägyptischen Museums, éd. W. Müller (Berlin: Akademie-Verlag, 1974), $283^{-285}$. La procédure devait être différente avant et après cette période. On peut imaginer que les contribuables ne recevaient pas de reçus ou alors des reçus sur papyrus, qui n' auraient pas été conservés.

23 Sur ces questions, voir Alain Delattre et Jean-Luc Fournet, "Le dossier des reçus de taxe thébains et la fiscalité en Égypte au début du viri ${ }^{\mathrm{e}}$ siècle," dans Coptica Argentoratensia: textes et documents de la troisième université d'été de papyrologie copte (Strasbourg, 18-25 
l'apparition des reçus de taxe sur ostraca à cette période reste donc ouverte. Par ailleurs, à côté des "garanties par Dieu", de véritables laissez-passer ont été émis dans la région, sans doute à la même époque.

Les fouilles belges dans la nécropole thébaine ont mis au jour, dans la tombe numéro 29, sur la colline de Cheick Abd el-Gournah, les restes d'un habitat monastique $^{24}$. La tombe fut occupée successivement par plusieurs personnages, dont le mieux connu, Frange, exerçait les professions de tisserand, de copiste et de relieur. Ses archives ont été découvertes à l' intérieur d' une faille au-dessus du puits funéraire, tandis que son métier à tisser a été mis au jour dans la cour et que des objets relatifs à son activité de relieur et de copiste ont été trouvés dans la tombe. Parmi ces derniers, on peut mentionner des calames, des restes de reliures et de fragments de papyrus destinés à être collés ensemble en une sorte de papier mâché, qui forme le plat des reliures. La plupart de ces documents sont des laissez-passer, comme l'a mis en évidence Anne Boud'hors ${ }^{25}$. Ils forment un corpus d'une cinquantaine de pièces, qui présentent un formulaire assez souple. Tous les textes sont adressés à deux personnes: Halakotse et Jôhannês. Le premier est le destinataire de trente exemplaires, Johannes de trois seulement. L' examen du dossier suggère que les deux responsables sont contemporains et non successifs: les documents semblent en effet avoir été écrits dans un laps de temps assez court. Plusieurs papyrus ont été retrouvés soigneusement roulés et pliés, avec un petit sceau en argile à l'intérieur. Les documents étaient donc à l'origine scellés, mais ont été ouverts et ensuite refermés.

Les textes commencent usuellement par la formule оү(0) мгк(, "veuille laisser (passer)", inconnue ailleurs dans la documentation. En voici un exemple $^{26}$.

juillet 2010) (P.Stras.Copt.), éds. Anne Boud'hors, Alain Delattre, Catherine Louis et Tonio Sebastian Richter (Paris: de Boccard, 2014), 209-244.

24 Les fouilles de l'Université libre de Bruxelles, commencées en 1997 par Roland Tefnin, sont dirigées depuis 2007 par Laurent Bavay.

25 Anne Boud'hors, "L'apport de papyrus postérieurs à la conquête arabe pour la datation des ostraca coptes de la tombe тт29," dans From al-Andalus to Khurasan:Documents from the Medieval Muslim World, éds. Petra M. Sijpesteijn, Lennart Sundelin, Sofía Torallas Tovar et Amalia Zomeño (Leiden: Brill, 2007), 115-129. Le dossier complet sera étudié par Anne Boud'hors et moi-même.

26 Boud'hors, “L' apport de papyrus postérieurs," no. 1. 
† Veuille laisser Chenoute (aller) au sud, car il est originaire du nome d'Ermont, qu'il aille chez lui. Du reste, ne l'empêche pas. Écrit au mois de Tybi, le 2o. $\dagger$ (Adresse au verso) À remettre à Halakotse de la part de Petros. $\dagger$

L'examen de ces documents permet de supposer l'existence d'un poste de contrôle, d'un checkpoint, au nord de Djême (plusieurs textes mentionnent qu' il s' agit d'aller "au sud, à Djême"). Halakotse et Jôhannês devaient être les responsables de ce poste. La mention du nome d'Hermonthis pourrait suggérer que les laissez-passer concernaient un déplacement entre deux nomes limitrophes.

Un deuxième document, inédit, le $\mathrm{n}^{\circ}$ d'inv. 295511, est plus explicite à ce sujet.

À remettre à Jôhannês de la part de Pehêu. Veuille laisser passer Petros et Mathias (ainsi que sa femme) au sud, avec leur matériel, qu' ils le vendent, et qu' ils me donnent l'argent. Tybi, le $20 . \dagger$

Petros et Mathias, ainsi que sa femme, mentionnée supra lineam, sont autorisés à aller au sud avec leurs objets pour les vendre. On peut imaginer que ce sont des artisans qui se rendent dans la ville de Djême vendre leur production. La raison du laissez-passer est ici précisée: il s' agissait de donner de l' argent, sans doute au titre du paiement des taxes, à l' expéditeur, Pehêu, probablement, à en juger par d' autres documents, un responsable local.

Ce type de document a selon toute vraisemblance été utilisé pendant une très courte période ${ }^{27}$, ce qui explique sans doute que les fouilles récentes en ont livré les premiers exemplaires connus ${ }^{28}$.

\section{Un nouveau témoin relatif à la circulation des personnes}

Le dossier des laissez-passer de la tombe thébaine 29 a connu récemment un intéressant développement. Lors du dégagement de la тT 29, une partie de la façade d'une nouvelle tombe est apparue aux archéologues. Il s' agit de la

27 Seuls quelques documents sont datés: ils le sont du mois de Tybi, d' une deuxième année de l'indiction.

28 Le fait est en effet étonnant, puisque la documentation thébaine est bien connue: environ la moitié des papyrus et ostraca coptes publiés, soit 4300 textes sur 8400 , provient de la région thébaine. 
tombe $\mathrm{C}_{3}$, une tombe thébaine déjà mentionnée dans la littérature scientifique au XIX ${ }^{e}$ siècle, mais dont la localisation n'était plus connue. Le dégagement de la cour a permis de découvrir de nombreux ostraca, mais aussi des structures chrétiennes, fort bien conservées, notamment une petite pièce comprenant deux lits. Un anachorète, et sans doute son disciple, ont vécu là, peut-être en même temps que Frange, qui occupait la tombe voisine ${ }^{29}$.

Le 3 février 2009, une série d'ostraca a été découverte, parmi lesquels ce texte-ci, à priori quelque peu énigmatique $(0.7012 \mathrm{~A}+\mathrm{B})^{30}$.

$\dagger$ †vant toute chose, nous t' écrivons en saluant ta paternité. Ensuite, nous t'informons que puisque tu as ... tu nous as écrit: "je veux envoyer cet homme à l'intérieur", maintenant, tout homme que tu veux, envoie-le à l' intérieur. Rien ne lui arrivera. Non seulement celui-là, mais aussi tout homme qui le veut, qu'il vienne à l' intérieur pour son travail (?). Aucun homme n'empêche un autre homme. Salut. † Donne à notre père Hellô, fils de Psôch, de la part d'Isak et de Jôannês. †

Le document a été écrit par Jôannês et Isak, dont les fonctions ne sont pas indiquées. Les deux noms sont courants dans la région thébaine, mais le ton officiel du texte permet de proposer de les identifier avec deux lachanes de Djême, attestés ensemble dans P.KRU 38, 7-10 (daté du 26 février 738 (31 $^{31}$ : NTIMIOTdTOC

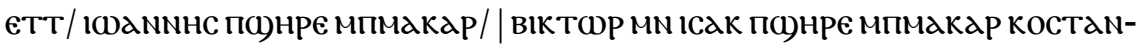

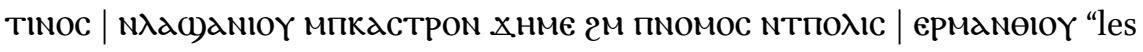
très honorés Jôannês, fils du défunt Biktôr, et Isak, fils du défunt Kostantinos, les lachanes du castrum de Djême, dans le nome de la ville d'Ermont". On notera que Frange apparaît également dans ce document (l. 69: ечрдмгe), ce qui confirme la proximité chronologique entre l'ostracon et le dossier de la TT 29, donc des laissez-passer.

Les lachanes écrivent à Hellô, fils de Psôch, un personnage qui n' est pas connu par ailleurs, mais qui pourrait être le voisin de Frange. Ils font part d'une précédente lettre de Hellô et lui indiquent que les déplacements sont autorisés et qu'il peut envoyer toute personne €20Yw, "à l' intérieur", une expression dont le sens n' est pas très clair. L'ostracon apporte ainsi des informations

29 Laurent Bavay, "La tombe perdue du substitut du chancelier, Amenhotep: données nouvelles sur l' organisation spatiale de la nécropole thébaine," Bulletin de la Société française d'Égyptologie 177-178 (2010): 28-30.

30 Depuis 2009, je suis chargé de l'étude et de la publication des documents écrits découverts lors des fouilles de la tombe $\mathrm{C} 3$.

31 Cf. aussi Till, Datierung und Prosopographie, 235. 
importantes sur les diverses limitations à la liberté de circulation dans la région thébaine au VIII ${ }^{\mathrm{e}}$ siècle: il nous indique que les entraves à la libre circulation n'étaient pas permanentes.

Reste à déterminer où se situe cet "intérieur" où l' on peut désormais envoyer des gens en toute quiétude. Anne Boud'hors et Chantal Heurtel ont montré que, dans plusieurs documents de tel la TT 29, l' expression "envoyer à l'intérieur" fait vraisemblablement référence à une clôture, réelle ou symbolique, délimitant l'habitat monastique ${ }^{32}$. Mais si l'ostracon est bien écrit par des lachanes de Djême et adressé à quelqu' un de la "sainte montagne", il serait plus logique de comprendre qu'il est question d' envoyer des gens à Djême même. On sait en effet que les déplacements entre Djême et la colline de Gourna étaient problématiques ${ }^{33}$. L'adverbe ęoY $\mathrm{N}$ serait alors à comprendre en référence $\mathrm{au}(\mathrm{x})$ locuteur(s) et l' "intérieur" dans la lettre renverrait à la ville plutôt qu' au monastère. Le checkpoint de Djême se situerait donc entre la ville et la sainte montagne. On pourrait l' imaginer à la porte de la ville, ou au contraire plus près de la colline de Cheick Abd el-Gournah.

On notera que dans $O$. Frange 9, le moine demande s' il y a un marchand d' huile à Djême ${ }^{34}$. La question semble indiquer que Frange connaît mal Djême, pourtant située à moins d'un kilomètre de sa cellule. On le voit par contre en étroit contact avec son village natal, Petemout, situé sur l' autre rive. Faut-il voir là une conséquence de ce barrage qui isolait, pendant un temps, la colline de Cheick Abd el-Gournah de la ville de Djême?

32 Anne Boud'hors et Chantal Heurtel, Les ostraca coptes de la TT 29:Autour du moine Frangé (Bruxelles: CReA Patrimoine, 2010), 16-18.

33 Le dossier des laissez-passer de la тT 29, par exemple, concerne essentiellement des déplacements de personnes qui veulent se rendre à Djême. Sur le sujet, on se reportera aussi à Lili Aït-Kaci, Anne Boud'hors et Chantal Heurtel, "Aller au nord, aller au sud, traverser le fleuve: Circulation et échanges au vin ${ }^{\mathrm{e}}$ siècle dans la région thébaine," dans Thèbes aux 101 portes: mélanges à la mémoire de Roland Tefnin, éds. Eugène Warwembol et Valérie Angenot (Turnhout: Association Égyptologique Reine Élisabeth, 2010), 1-9.

34 L. 6-13: "Ayez la bonté, si quelqu' un passe chez vous qui soit rattaché à Djême, demandezlui s'il y a un homme qui vend de l'huile à Djême pour que j' envoie en chercher un peu et que j' en aie pour la fête" (trad. Boud'hors et Heurtel). Cf. aussi Aït-Kaci, Boud'hors et Heurtel, "Aller au nord." 
Les sources présentées ici illustrent différentes facettes des mécanismes de contrôle de la population mis en place par l'administration arabe au début du VIII ${ }^{\mathrm{e}}$ siècle. Les allusions aux taxes dans de nombreux documents montrent que ce contrôle avait pour but d'assurer une meilleure perception des impôts et une application efficace des corvées et réquisitions, en évitant notamment la fuite des paysans ${ }^{35}$.

Trois types de documents, à peu près contemporains, ont pu servir de saufconduits. Ils possèdent chacun un formulaire spécifique et étaient utilisés, selon toute vraisemblance, dans des situations différentes.

1. Les sauf-conduits arabes (et peut-être aussi bilingues), étaient des documents officiels, nécessaires pour entreprendre de longs voyages entre différentes régions d'Égypte. Ils étaient émis par l'administration, qui exigeait des garants ${ }^{36}$.

2. Les "lettres de protection", 入огос мпмоүте, permettaient de circuler sur de courtes distances; elles n'étaient pas à proprement parler des saufconduits, mais plutôt des garanties, émises par les administrations locales, qui se portaient ainsi caution de la solvabilité du porteur du document $^{37}$.

3. Enfin, les "laissez-passer" de la tombe thébaine 29 permettaient de franchir un poste de contrôle temporaire situé au nord de la ville de Djême, peut-être en venant d' un autre nome ${ }^{38}$.

Si les deux premiers types de documents sont attestés dans diverses régions de l'Égypte, il n' en va pas de même du troisième, qui est très local et qui n'a été utilisé, pour autant qu' on puisse en juger, que durant une période fort courte. La découverte de l'ostracon de la tombe c3 montre par ailleurs qu'il y avait des restrictions particulières à la libre circulation dans la région thébaine, mais qu' elles pouvaient être allégées ou renforcées.

Je propose donc de voir dans les laissez-passer de тт 29 et dans l' ostracon de $\mathrm{C} 3$ les témoins d'un événement spécifique à la région thébaine, que le hasard de la découverte et de la conservation des sources permet de mettre en évidence,

35 Cf. les lettres de protection liées à des reçus de taxe et la mention des impôts acquittés dans P.CLT 3 ou dans les garanties de Saqqarah. Sur les corvées dans le cadre du cursus, cf. Trombley, "Sawīrus Ibn Al-Muqaffac," 202-204, 215-216.

$36 \quad$ Voir plus haut les sections 2-4.

$37 \quad$ Voir plus haut la section 5 .

38 Voir plus haut les sections $6-7$. 
mais qui s' intègre dans un contexte plus large. La région semble en effet avoir connu plusieurs bouleversements entre la fin du VII ${ }^{\mathrm{e}}$ siècle et la première moitié du VIII ${ }^{\mathrm{e}}$ siècle. En raison de la pression fiscale, des troubles ont éclaté dans le pays ${ }^{39}$, et notamment dans la région thébaine, comme le montre $s B$ III 7240 , daté du 17 octobre $697^{40}$. Dans ce document, le duc Flavius Atias s' adresse aux moines de la communauté de la Jarre (le même établissement que dans P.CLT 3 cité plus haut) et fait état d' une révolte durant laquelle les moines n' ont pas voulu payer les impôts ${ }^{41}$. Atias mentionne un document émis par un de ses prédécesseurs, qui autorise les moines à habiter le monastère, pour autant qu' ils payent les impôts, et le duc confirme et renouvelle l' autorisation. Mais les problèmes de perception des taxes ont certainement continué: les décalages entre date de paiement et année d'imposition ainsi que les fractionnements des paiements dans les reçus de taxe thébains l'attestent. Il semble même qu' une contribution punitive (le $\pi \rho o ́ \sigma \tau \mu o v)$ ait été levée en mars $720^{42}$.

Dans le contexte ainsi brossé, les différentes sources présentées ici sont autant d'éclairages sur la situation de l'Égypte au début du viII ${ }^{\mathrm{e}}$ siècle. Les documents thébains nous font connaître un peu mieux les mécanismes de contrôle de la population que pouvaient mettre en place les autorités, non seulement dans la région, mais sans doute aussi dans d'autres localités et d' autres provinces de l'Empire omeyyade.

\section{Bibliographie}

Aït-Kaci, Lili, Anne Boud'hors et Chantal Heurtel. "Aller au nord, aller au sud, traverser le fleuve: Circulation et échanges au viII ${ }^{\mathrm{e}}$ siècle dans la région thébaine." Dans Thèbes aux 101 portes: Mélanges à la mémoire de Roland Tefnin, édité par Eugène Warwembol et Valérie Angenot, 1-9. Turnhout: Association Égyptologique Reine Élisabeth, 2010.

Antoniak, Iwona. "Preliminary Remarks on the Coptic Ostraca from the Seasons 2003 and 2004." Polish Archaeology in the Mediterranean 16 (2005): 244-247.

39 Cf. Petra M. Sijpesteijn, "Landholding Patterns in Early Islamic Egypt," Journal of Agrarian Change 9, no. 1 (2009): 129 .

40 Cf. $B L$ VIII, $326-327$.

41 Sur le personnage, cf. $C P R$ VIII $72-84$ (qui, pour l' essentiel, proviennent du nome arsinoïte et datent de la fin du viI ${ }^{\mathrm{e}}$ siècle).

42 Cf. Delattre et Fournet, "Des reçus de taxe thébains," 220. Plusieurs autres contributions exceptionnelles ont été levées dans les années $720-726$. 
Bavay, Laurent. "La tombe perdue du substitut du chancelier, Amenhotep: données nouvelles sur l' organisation spatiale de la nécropole thébaine." Bulletin de la Société française d'Égyptologie 177-178 (2010): 23-43.

Boud'hors, Anne. "L' apport de papyrus postérieurs à la conquête arabe pour la datation des ostraca coptes de la tombe TT29." Dans From al-Andalus to Khurasan:Documents From the Medieval Muslim World, édité par Petra M. Sijpesteijn, Lennart Sundelin, Sofía Torallas Tovar et Amalia Zomeño, 115-129. Leiden: Brill, 2007.

Boud'hors, Anne, et Chantal Heurtel. Les ostraca coptes de la TT 29: autour du moine Frangé. Bruxelles: CReA Patrimoine, 2010.

Cromwell, Jennifer. "Aristophanes Son of Johannes: An Eighth-Century Bilingual Scribe? A Study of Graphic Bilingualism." Dans The Multilingual Experience in Egypt, from the Ptolemies to the Abbasids, édité par Arietta Papaconstantinou, 221-232. Farnham: Ashgate, 2010.

Crum, Walter E. A Coptic Dictionary. Oxford: Clarendon Press, 1939.

Delattre, Alain. "Les 'lettres de protection' coptes." Dans Akten des 23. Internationalen Papyrologenkongresses. Wien, 22.-28. Juli 2001, édité par Bernhard Palme, 173-178. Vienne: Verlag der Österreichischen Akademie der Wissenschaften, 2007.

Delattre, Alain, et Jean-Luc Fournet. "Le dossier des reçus de taxe thébains et la fiscalité en Égypte au début du VIII ${ }^{\mathrm{e}}$ siècle." Dans Coptica Argentoratensia: textes et documents de la troisième université d'été de papyrologie copte (Strasbourg, 18-25 juillet 2010) (P.Stras.Copt.), édité par Anne Boud'hors, Catherine Louis, Alain Delattre et Tonio Sebastian Richter, 209-244. Paris: de Boccard, 2014.

Delattre, Alain, et Rosario Pintaudi. "Les archives de Paule, fils de Petros, de la rue du Sauveur." Dans Antinoupolis II I, édité par Rosario Pintaudi. Florence: Istituto Papirologico "G. Vitelli," à paraître.

Diem, W. "Einige frühe amtliche Urkunden aus der Sammlung Papyrus Erhzerzog Rainer (Wien)." Le Muséon 97 (1984): 109-158 (= PERF 598).

Evetts, Basil. "History of the Patriarchs of the Coptic Church of Alexandria. Agathon to Michael III (766).” Patrologia Orientalis 5, no. 1 (1910): 1-215.

Frantz-Murphy, Gladys. Arabic Agricultural Leases and Tax Receipts from Egypt, 148427 A.H./765-1035A.D. Vienna: Hollinek, 2001.

Frantz-Murphy, Gladys. "Identity and Security in the Mediterranean World ca. AD 640ca. 1517." Dans XXvth International Congress of Papyrology. Ann Arbor, July 29-August 4, 2007, édité par Traianos Gagos, 253-264. American Studies in Papyrology. Ann Arbor: University of Michigan Press, 2010.

Kahle, Paul E. "Zu den koptischen Steuerquittungen." Dans Festschrift zum 150-jährigen Bestehen des Berliner Ägyptischen Museums, édité par W. Müller, 283-285. Berlin: Akademie-Verlag, 1974.

Mitthof, Fritz. "Urkundenreferat 2007 (2. Teil).” Archiv für Papyrusforschung 55, no. 1 (2009): 149-170. 
Pilette, Perrine, et Naïm Vanthieghem. "Un nouveau sauf-conduit du monastère d' Apa Jeremias à Saqqara?" Bulletin of the American Society of Papyrologists 53 (2016): 233238.

Rāg̀ib, Yūsuf. "Sauf-conduits d'Égypte omeyyade et abbasside." Annales Islamologiques 31 (1997): 143-168.

Schaten, Sofia. "Reiseformalitäten im frühislamischen Ägypten." Bulletin de la Société d'Archéologie Copte 37 (1998): 91-10o.

Sijpesteijn, Petra M. "Landholding Patterns in Early Islamic Egypt." Journal of Agrarian Change 9, no. 1 (2009): 120-133.

Sijpesteijn, Petra M. "Coptic and Arabic Papyri from Deir al Balāizah." Dans Actes du $26^{e}$ Congrès international de papyrologie, édité par Paul Schubert, 707-713. Genève: Droz, 2012.

Sijpesteijn, Petra M. Shaping a Muslim State: The World of a Mid-Eighth-Century Egyptian Official. Oxford: Oxford University Press, 2013.

Till, Walter C. Datierung und Prosopographie der koptischen Urkunden aus Theben. Vienne: Österreichische Akademie der Wissenschaften, 1962.

Torallas Tovar, Sofía, et Amalia Zomeño. "El control de la población en el Egipto pre y protoárabe." Dans Historiografía y representaciones III: estudios sobre las fuentes de la conquista islámica, édité par Luis A. García Moreno, Esther Sánchez Medina et Lidia Fernández Fonfría, 6o9-624. Madrid: Real Academia de la Historia, 2009.

Trombley, Frank R. "Sawīrus Ibn Al-Muqaffa' and the Christians of Umayyad Egypt: War and Society in Documentary Context." Dans Papyrology and the History of Islamic Egypt, édité par Petra M. Sijpesteijn et Lennart Sundelin, 199-226. Leiden: Brill, 2004.

Vanthieghem, Naïm. "Le plus ancien sauf-conduit arabe." Der Islam 91, no. 2 (2014): 266271. 